\title{
A Viable Upload Acceleration Service for Mobile Devices
}

\author{
Yan Pu, Akihiro Nakao \\ Graduate School of Interdisciplinary Information Studies \\ The University of Tokyo \\ 7-3-1 Hongo, Bunkyo-Ku,Tokyo, Japan \\ \{yan,nakao\}@nakao-lab.org
}

\begin{abstract}
As smart phones and mobile devices are proliferating, the traffic volume generated by them is growing drastically these days [1]. Due to these devices are often connected to WiFi access points (APs) for the first/last mile accesses to the Internet, WiFI APs accommodate large traffic to and from these mobile devices. We observe that while downloading large content onto such mobile devices is often aided by caches placed at and near the WiFi APs, uploading has rarely been considered. This paper proposes a viable uploading acceleration service on WiFi APs. To the best of our knowledge, this paper is the first to introduce the design of the framework of upload acceleration for mobile devices. We also implement our first prototype system on top of virtualized WiFi APs and evaluate the reduction of upload time of video files via our prototype.
\end{abstract}

\section{Categories and Subject Descriptors}

C.2.1 [Network Architecture and Design]: :Wireless Communication

\section{General Terms}

Design, Performance, Experimentation

\section{Keywords}

Wireless, Upload, Virtualization, Cache, Mobile Clients

\section{INTRODUCTION}

Recently, many popular cloud computing applications and services such as Youtube and Facebook have been developed on mobile devices so that users may directly upload and share photos, video, documents (so-called

Permission to make digital or hard copies of all or part of this work for personal or classroom use is granted without fee provided that copies are not made or distributed for profit or commercial advantage and that copies bear this notice and the full citation on the first page. To copy otherwise, to republish, to post on servers or to redistribute to lists, requires prior specific permission and/or a fee.

ACM CoNEXT Student Workshop, December 6, 2011, Tokyo, Japan.

Copyright 2011 ACM 978-1-4503-1042-0/11/0012 ...\$10.00.
User-Generated-Content (UGC)) to data-centers. However, compared with download, the throughput of upload is not satisfactory due to the following possible reasons: First, a network operator often configures asymmetric connectivities for uplink and downlink as observed in ADSL, for example. Second, as mentioned, caches placed at or near the WiFi APs are also helpful for accelerating the data download.

Since minimizing power consumption is always the center of mobile computing, it is crucial to quickly complete data transmission so that mobile devices may not stay connected to APs long, thus, may become highly mobile, and also may save their battery power. Now that uploading relatively large content such as video clips taken by mobile devices becomes popular, it is important to accelerate the upload performance for mobile devices.

This paper proposes a system design to enable upload caching to accelerate upload communication, taking WiFi access points as an example. We first describe the system design for our service and then introduce our prototype system and evaluate its performance for reducing latency in uploading large user-generatedcontent (UGC).

\section{SYSTEM DESIGN FOR UPLOAD ACCEL- ERATION SERVICE}

We design our system for upload acceleration service on WiFi APs as shown in Figure 1.

\subsection{Wireless Extension by Virtualized WiFi Ac- cess Point}

In our system each WiFi AP holds multiple VMs and Open vSwitches [2] so that we can selectively redirect the target flows and enable upload acceleration only to them, as shown in Figure 1.

\subsection{Design and Deployment of Service}

The upload acceleration service is designed to be deployed inside a VM as an application-layer service. In this way, it can be easily programmed and enabled on top of the existing protocols. To achieve the acceleration of data uploading, we ensure that packets may 


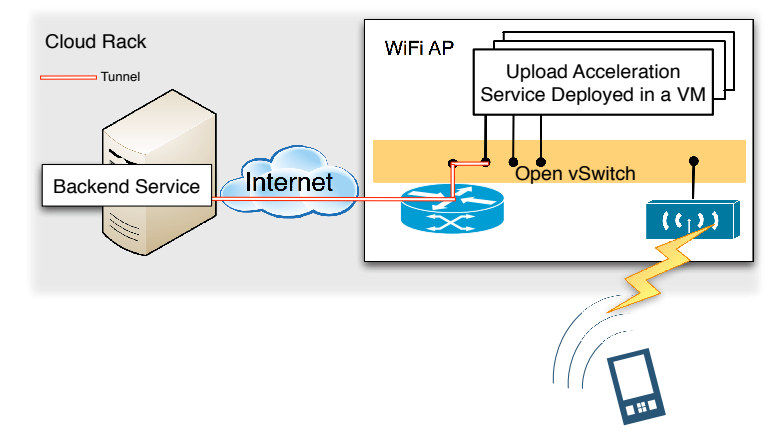

Figure 1: System Design for Upload Acceleration Service

be first forwarded to the service deployed in a VM and stored there temporarily. Then, after all the data is received in the VM, a mobile client can close its connection for uploading. Due to the direct link between a mobile client and the AP usually has lower jitter, more stable throughput can be achieved and the average throughput also tends to be higher compared with the throughput on the whole link.

A user is most likely to access the most recently uploaded data. For example, if a user uploads a video clip, he/she may want to review it immediately. Therefore, it makes sense to keep the cache of the most recent uploaded data.

Additionally, if we combine this system design with live-migration of VMs, we can migrate the VM with upload acceleration service wherever a user may go. For example, CloudRack [3] can preserve the virtual network topology after VM migration, one possible implementation of this service is to combine CloudRack with our upload acceleration service proposed in this paper.

\section{PROTOTYPE IMPLEMENTATION AND EVALUATION}

To validate our proposal, we have developed a preliminary prototype of our proposed service. We extend basic CloudRack architecture with VMs on top of a WiFi AP. We apply HostAP driver in the Linux 2.6.35 kernel with Open vSwitch enabled.

For our evaluation, we prepare a backend web service that receives videos and photos from a user's iPhone. The web service allows the user to review all the uploaded data via a web browser. The front-end upload acceleration service is also implemented as a web service that receives the data from the user and caches and forward the data to the backend service.

Based on our prototype implementation, we take videos of different length and send them to the backend VM with or without the acceleration service applied. We record the uploading time of these videos. The result is shown in Figure 2.

The results show that the transmission time is obvi-

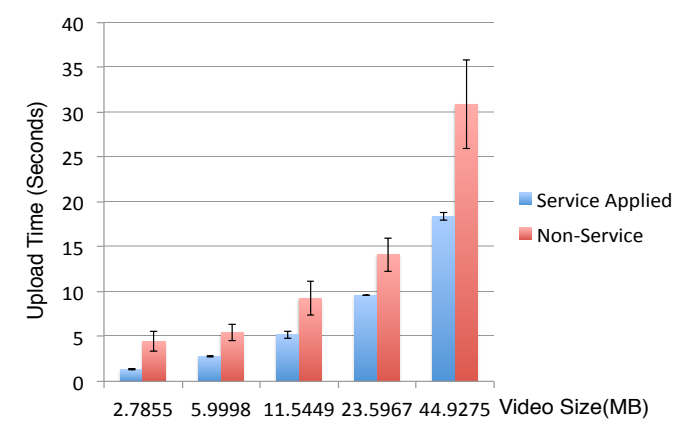

Figure 2: Results of Upload Acceleration Experiments

Table 1: Standard Deviation of Upload Time

\begin{tabular}{|l|r|r|}
\hline File Size(MB) & Accelerated(Sec.) & Non-Service(Sec.) \\
\hline 2.7855 & 0.1471 & 2.4382 \\
5.9998 & 0.1994 & 2.1217 \\
11.5449 & 0.8734 & 4.2837 \\
23.5967 & 0.0748 & 4.1980 \\
44.9275 & 0.9139 & 11.0462 \\
\hline
\end{tabular}

ously reduced when the upload acceleration service is applied. Besides, the throughput is also more stable as the standard deviation of upload transmission time shown in Table 1. The results show that our proposed approach successfully reduces the transmission time and makes it stable.

\section{CONCLUSION AND FUTURE WORK}

We propose a viable upload acceleration service in this paper. Our service is practical to work on the virtualized network architecture CloudRack as an extension. The current cloud services can be easily modified for enabling our service. Our experiments using prototype implementation clearly show that the upload time is considerably reduced by this acceleration service.

For future work, we plan to implement our service in the network layer instead of application layer in order to achieve higher throughput $\mathrm{i}$ and also to make the service transparent to service providers.

\section{REFERENCES}

[1] comScore. Smartphones and tablets drive nearly 7 percent of total u.s. digital traffic. http://www. comscore.com/.

[2] B. Pfaff and et al. e.a.: Extending networking into the virtualization layer. In In: 8th ACM Workshop on Hot Topics inNetworks (HotNets-VIII).New YorkCity, NY (October 2009.

[3] P. Yan and et al. Cloud rack: Enhanced virtual topology migration approach with open vswitch. In Information Networking (ICOIN), 2011 International Conference on, jan. 2011. 